\section{Negative Staining: Historical Background and Technical Development}

$\mathrm{J}$. Robin Harris, Institute of Zoology, University of Mainz

The following is a chapter from the new RMS Microscopy Handbook "Negative Staining and Cryoelectron Microscopy." The handbook is available from Microscopy Today at a price of $\$ 40.00$ pius $\$ 5.00 \mathrm{~S} \& \mathrm{H}$.

There is a considerable body of information of a technical and review nature on negative staining elsewhere in the literature (Bremner et al., 1992; Harris and Horne, 1991, 1994; Hayat and Miller, 1990; Holzenburg, 1988; Horne, 1991; Nermut, 1991; Spiess et al., 1987; Valentine and Horne, 1962); therefore, I shall attempt to avoid undue repetition and present the current laboratory approaches with which I am personally familiar and have achieved some technical success. After considering the range of procedures necessary for the production of carbon and carbon-plastic support films (sometimes termed substrates) emphasis will be placed upon the droplet negative staining technique (Harris and Agutter, 1970; Harris and Horne, 1991). I was introduced to this approach by Ennio Lucio Benedetti in his laboratory, at the start of my doctoral studies in 1966 (Anderson, 1966; Benedetti and Emmelot, 1965, 1968) and with minor variations it is still useful for many biological applications. Secondary to this approach, but with perhaps considerably greater scope for further technical development, are the floating method and the negative staining - carbon film (NS - CF) procedures. Actually, both of these are 'floating' variants of the negative staining technique and have their roots in the mica - carbon film production procedure (Section 3.1), using the inherent property that a freshly released floating layer of carbon has a strong adsorptive and stabilization capacity for proteins and viruses (Valentine et al., 1966). When developed further by Ivonne Pasquali-Ronchetti and Bob Horne in the early 1970 s by spreading virus suspensions in the presence of ammonium molybdate on a mica

\section{COSTEFHECTIVE EM MAGNETIC SHUILIDINGL}

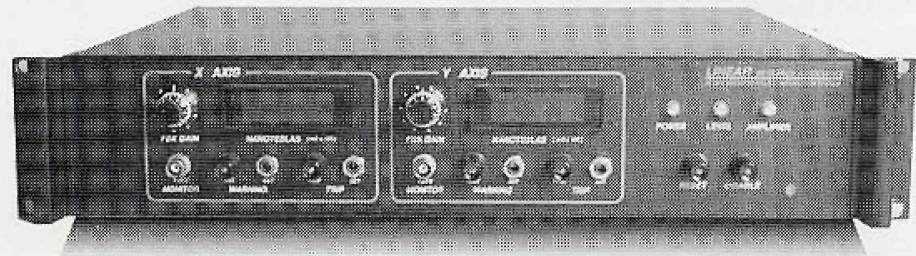

EM sites often require supplemental magnetic shielding to achieve full resolution. LINEAR RESEARCH ASSOCIATES' wideband EMFC$A C$ electronic active-shielding systems dramatically reduce magnetic fields radiating from nearby a.c. power wiring, ground loops, transformers and related sources. EMFC-QDC extended-range models additionally suppress low-frequency magnetic disturbances caused by trains, subways, elevators and equipment such as MRI scanners.

EMFC active-shielding is highly recommended for cost-effective protection of sensitive FEG and PEELS installations.

Linear Research Associates' EMFC equipment is the highestperformance commercially available magnetic field compensation apparatus in the world. EMFC systems feature state-of-the-art engineeringtt and are U.S.-manufactured to exacting standards. Superior performance and reliability are guaranteed!

Call or fax LRA for complete EMFC-series information. We will also gladly assist with any questions you may have regarding site survey, engineering or EMF issues.

\section{LINEAR RESEARCH ASSOCIATES}

surface before carbon coating, this led to the production of ordered arrays on viruses attached to the carbon film, which subsequently could be negatively $\bar{B}$ stained (for details see Section 3.3); thus the proposal of the rather verbose terminology 'negative staining - carbon film technique'. My own interest in the NS CF procedure for the production of ordered arrays and 2-D crystals of protein molecules commenced soon after the initial description of this approach (Hornen and Pasquali-Ronchetti, 1974) and continues strongly to this day (Harris, 1991 ज्ञ Harris and Holzenburg, 1989, 1995).

The concept of negative staining as a light microscopical procedure that will enable an essentially transparent object to be rendered visible by surrounding if with a colored solution is rather old, but the transfer of this idea to thinly spread material for electron microscopy came only slowly. It is widely accepted that Bobz Horne was the first electron microscopist to really come to terms with the subject? He presented clear data showing that bacteriophages could be surrounded by af thin amorpyhous layer of air-dried sodium phosphotungstate or uranyl acetate which considerably reduced the structural flattening that occurred in the absence of stain (Brenner and Horne, 1959). Early attempts to positively stain protein molecules and viruses with low $\mathrm{pH}$ phosphotungstate also gave some indicatior of negative staining when the stain was in excess or incompletely washed away: but this was not established independently as a routine procedure (Hall, $1955^{\circ}$ Huxley and Zuban, 1960). Clearly, in this early developmental work it waso important to select heavy metal salts that air-dried to give an evenly spreach non-crystalline amorphous or vitreous glass-like electron-opaque or -dense layer surrounding and supporting (i.e., embedding) the biological particles.

Negative staining is believed to reduce the surface tension forces at the air-fluid interface that would otherwise produce considerable flattening of biological material on to a support film and there actual thickness of the dried stain layer is also likely to be rather important. The thickness of the dried negative stain will be determined by the concentration and the volume of fluid dried on to the support film. As indicated below (also Section 3.2) the thickness of the supporting layer of dried stain can be considerably increased by including a carbohydrate such as glucose or trehalose, which is also likely to protect protein structure during specimen drying. Ideally, negative stains should not interact with biological material in a 'positive' staining manner, neither must they cause any aggregation or precipitation, or indeed molecular dissociation (but see Sections 3.3 .2 and 3.5.6). A very limited number of heavy metal salts were found to be suitable as negative stains, and in all cases it was necessary to avoid the additional presence of sodium chloride, buffer salts and routine biochemical reagents such as urea, glycerol and sucrose, other than as trace quantities.

The most useful negative staining salts defined by the early investigators are uranyl acetate, uranyl formate, sodium/potassium phosphotungstate, sodium silicotungstate and ammonium molybdate. Other, lesser used salts will be mentioned below (Section 3.2.3). That these salts all have the property of drying as an amorphous layer does not necessarily mean that microcrystallites of stain are not present in this layer. Indeed, this situation and the possible lability and even mobility of such crystallites within the electron beam has been addressed (Unwin, 1974). Since the ability of any negative stain to reveal structural detail

Continued on Page 12

Call to request our new catalog! Quality E.M. supplies
"taylored" to fit your
application and budget!

M.E. TAYLOR ENGINEERING, INC. 21604 Gentry Lane • Brookeville, MD 20833

Phone: 1-301-774-6246 FAX: 1-301-774-6711

E-Mail: Metengr@aol.com

VISA AND MASTERCARD ACCEPTED! 


\section{If you thought Digital Microscopy was a thing of the future...}

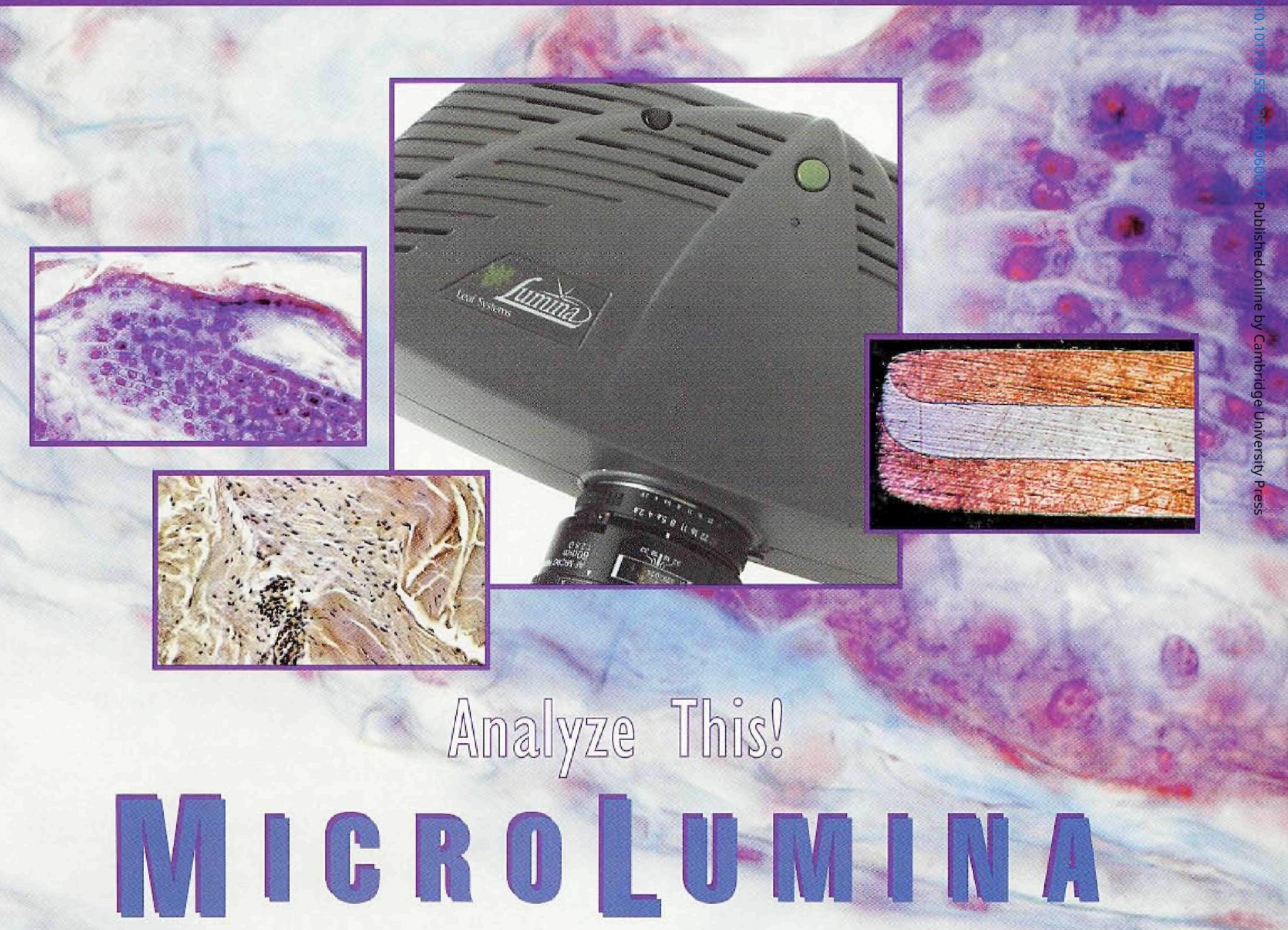

An Ultra Resolution Scanning Digital Camera that captures 36 bit images at up to $3380 \times 2700$ pixel resolution. Its high powered scanning is achieved through the use of a tri-linear $2700 \times 3$ coupled device (CCD) which captures images with exceptional quality in a single pass.

It produces images at approximately 30 times the resolution of video cameras, allowing enlargement of micrographs and micrography without pixelization. It can create files ranging in size from $100 \mathrm{~K}$ to a full resolution $26 \mathrm{MB}$ file in under one minute (for full frame, full resolution image) and it works directly with your Mac or PC without the need for a framegrabber.

The MicroLumina comes with our new EasyScan 1.0

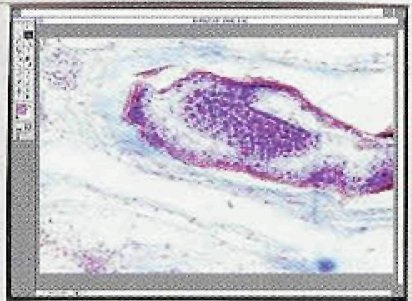

imaging software which features full field preview and prescan with image size controls, toning controls, automatic exposure, and manual exposure override.

EasyScan 1.0 offers flexibility and power

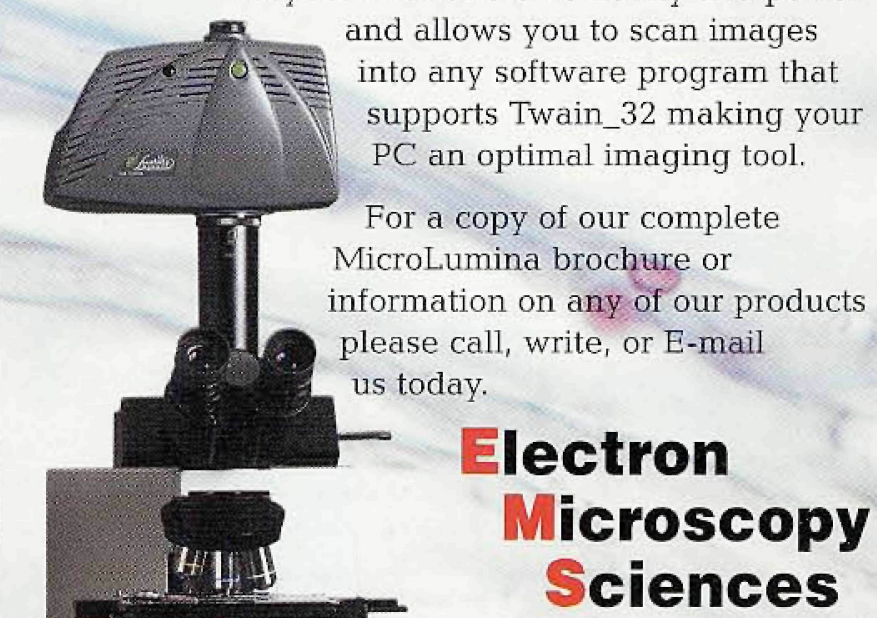

321 Morris Road - P.O. Box 251

Fort Washington, PA 19034

(215) $646-1566 \cdot(800) 523-5874$ Fax: (215) 646-8931

E-mail:sgkcck@aol.com http://www.emsdiasum.com 


\section{STAFF SCIENTIST}

Emest Orlando Lawrence Berkeley National Laboratory currently has an opportunity for a full-time Staff Scientist to work in its state-of-the-art National Center for Electron Microscopy (NCEM). The successful candidate will conduct materials science research as well as lead the development and operation of the In Situ Microscopy program.

This position requires a background in transmission electron microscopy. You must have experience in dynamic experimentation, specimen preparation and advanced microscopy techniques, including high-resolution imaging, high-voltage microscopy, convergent beam diffraction, micro-analytical techniques or computer image analysis/interpretation. A Ph.D. in the physical sciences is strongly preferred.

Please send resume and cover letter to: Lawrence Berkeley Laboratory, Staffing Office, Job \#MT/4891, One Cyclotron Road, MS 938A, Berkeley, CA 94720.
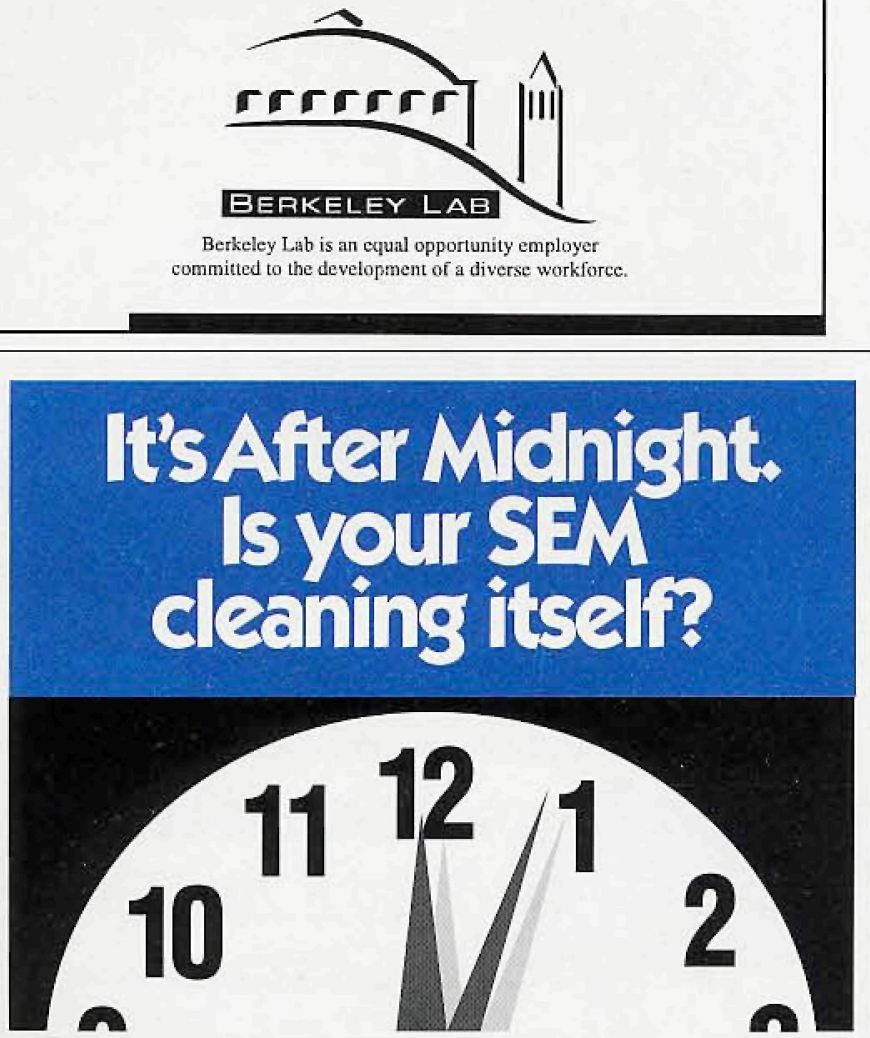

It would be if you had the SEM-CLEAN ${ }^{\top w}$ anti-contamination system. Overnight, the inexpensive SEM-CLEAN system uses a Nitrogen purge to clean your electron microscope. Its active cleaning action removes oils and other adsorbed contaminants from the walls of the chamber and carries them to the roughing pump. Result? Significant reductions in, even elimination of, sample-contamination, raster burns and oil buildup on EDS windows. Over 50 satisfied SEM-CLEAN users are our best references. For a list and complete details, call or write today.

\section{SEM-CLEAN"' Stops the Oil}

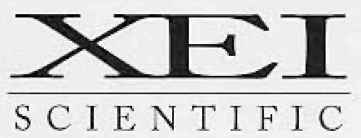

3124 Wessex Way, Redwood City, CA, U.S.A 94061 415-369-0133 - Fax 415-363-1659

\section{Negative Staining - continued from page 10}

must depend upon permeation of the staining cation or anion into aqueous cavities inside a biological particle, this could be limited by the size of such crystallites, but perhaps ultimately by the hydrated ionic diameter of the negative staining salt in solution. Thus, it is likely that negative staining will reveal primarily the outer surface and therefore the overall shape/replica or quaternary structure of a protein molecule, and be unable to reveal high resolution detail (i.e., better than c. $15 \dot{A}_{i}$ but see Kiselev et al, 1990, and Chapter 9). Certainly the presence of surface regions containing a number of hydrophobic amino acids and hydrophobic pockets within a protein are unlikely to be optimally negatively stained. Regions containing a net positive or net negative charge are likely to interact differently with anionic and cationic negative stains, respectively. For instance, the uranyl cation is known to bind to phosphate groups and also to carboxyl groups, whereas the molybdate anion is likely to sequester bound calcium and magnesium and may also interact directly with available positively charged amino groups. These more detailed considerations apart, the negative stains generally appear to behave in a similar manner, based upon the overall comparability of the electron optical images in different stains. Nevertheless, significant staining differences may occur (Woodcock and Baumeister, 1990). During the several decades since the establishment of the negative staining procedure numerous scientists have successfully utilized this approach for the study of a wide range of biological material. Due credit should be given to those working within the group of Wolfgang Baumeister (in Martinsried), those in the group of Erin van Bruggen (in Groningen) those with Joachim Frank (in New York) and Jose Carrascosa (in Madrid), and those in the groups of Ueli Aebi and Andreas Engel (in Brasel). Of the many individuals who have made a significant contribution over the years, the names of Linda Amos, Egbert Boekema, Roger Craig, Howard Egelman, Andeas Holzenburg, Kevin Leonard, Bill Massover, Milan Nermut and Alasdian Steven might be mentioned.

After a lengthy period of procedural stability and lack of progress, I feel that further technical development and a more critical assessment of the potential of negative staining is now required (Harris and Horne, 1994). The routine inclusion of a carbohydrate such as glucose or trehalose (Harris et al., 1995) along with negative stain, combined with low temperature TEM study, appears to provide some improvement of imaging. At the same time this carbohydrate-stain mixture creates a thicker amorphous layer within which protein molecules can more freely adopt varying orientations with respect to the carbon support film, with reduced adsorptive interaction and the likelihood of structural changes or restriction of stain access because of close apposition of protein and carbon. This thicker layer of stain and trehalose considerably reduces the undesirable flattening of tubular structures or other fragile structures such as liposomes (see Section 3.2 and Chapter 5). Trehalose has the unique ability to create vitreous/glass-like carbohydrate-water films in which the water is tightly bound (Green and Angell, 1989), which may explain its ubiquitous and extremely useful property as a biological protectant at extremes of temperature and dehydration, and to UV irradiation. Study of negatively stained specimens at low temperature in a specimen holder cooled by liquid nitrogen or liquid helium, although not new, has until recently (Harris et al., 1995) been used primarily by those investigating thin 3-D and 2-D protein crystals.

In the presence of negative stain and trehalose, or glucose, low temperature $\left(-170^{\circ} \mathrm{C}\right.$, or below) and low electron dose study is certainly desirable to obtain the best resolution from biological material (Kiselev et al., 1990). Nevertheless, with trehalose, room temperature studies can be performed providing the stain-carbohydrate layer is relatively thin and a low electron doses maintained, otherwise pronounced 'bubbling' can occur (see Section 4.3). Although in the early work in this area only combinations of uranyl acetate and glucose were used, it may be significant that more recent studies have also utilized ammonium molybdate together with glucose or trehalose. From theoretical considerations ammonium molybdate is likely to be a superior negative stain to uranyl acetate, since it is not generally known to impart any element of positive staining. It is likely that this approach can be extended to combinations of trehalose with any of the known anionic negative staining salts. 


\section{Where Else Can You Get...}

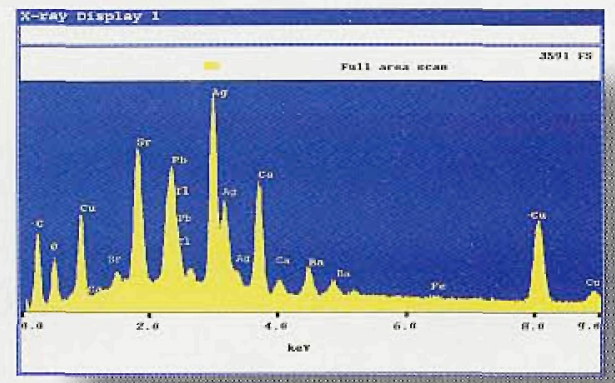

- Full Area Spectrum

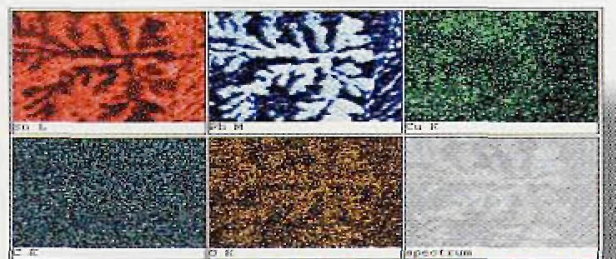

- A Map for Every Element

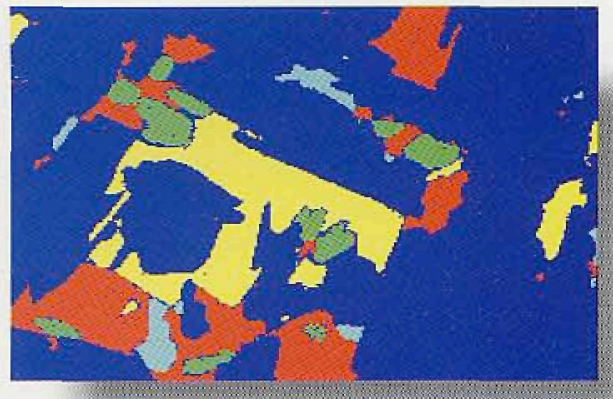

\section{- A Spectrum From Each Phase}

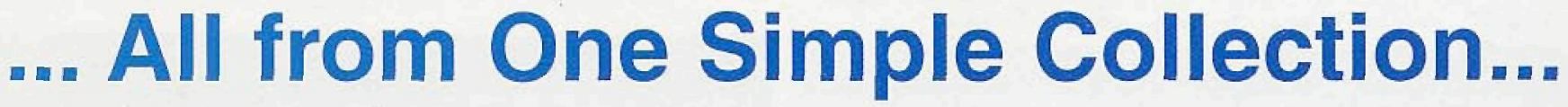

PGT introduces IMIX-PTS...Position Tagged Spectrometry. This new and revolutionary technique for X-ray microanalysis data acquisition features PGT's patented digital pulse processor. While the Electron Microscope rapidly scans the sample and acquires a high resolution electron image, $\mathrm{X}$ rays are processed and encoded with the specimen $(x, y)$ coordinate information. There is no need to perform separate collections for images, maps and spectral data or to decide beforehand what elements to map.

For more information contact PGT or visit us on the World Wide Web.

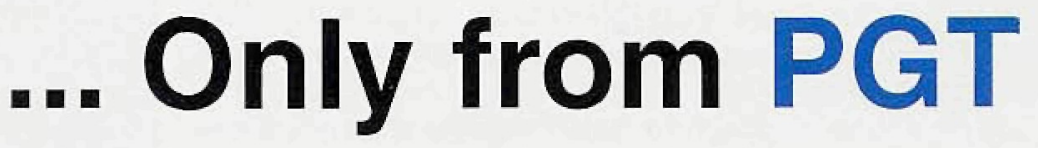

$\partial \square|=| 1200$ State Road P Princeton, NJ 08540 - Tel: (609) 924-7310 - Fax: (609) 924-1729 • Web Site: http://www.pgt.com 